\title{
Incentives for Ridesharing: A Case Study of Welfare and Traffic Congestion
}

\author{
Changle Song, ${ }^{1}$ Julien Monteil, ${ }^{2}$ Jean-Luc Ygnace, ${ }^{3}$ and David Rey $\mathbb{D}^{1,3}$ \\ ${ }^{1}$ School of Civil and Environmental Engineering, UNSW Sydney, Sydney, 2052 NSW, Australia \\ ${ }^{2}$ IBM Research, Ireland Lab, Dublin, Ireland \\ ${ }^{3}$ University of Gustave Eiffel, University of Lyon, IFSTTAR, ENTPE, LICIT UMR T9401, Lyon, F-69675, France
}

Correspondence should be addressed to David Rey; d.rey@unsw.edu.au

Received 18 December 2020; Revised 28 March 2021; Accepted 28 May 2021; Published 7 June 2021

Academic Editor: Dilum Dissanayake

Copyright ( $\odot 2021$ Changle Song et al. This is an open access article distributed under the Creative Commons Attribution License, which permits unrestricted use, distribution, and reproduction in any medium, provided the original work is properly cited.

Traffic congestion is largely due to the high proportion of solo drivers during peak hours. Ridesharing, in the sense of carpooling, has emerged as a travel mode with the potential to reduce congestion by increasing the average vehicle occupancy rates and reduce the number of vehicles during commuting periods. In this study, we propose a simulation-based optimization framework to explore the potential of subsidizing ridesharing users, drivers, and riders, so as to improve social welfare and reduce congestion. We focus our attention on a realistic case study representative of the morning commute on Sydney's M4 Motorway in Australia. We synthesize a network model and travel demand data from open data sources and use a multinomial logistic model to capture users' preferences across different travel roles, including solo drivers, ridesharing drivers, ridesharing passengers, and a reserve option that does not contribute to congestion on the freeway network. We use a link transmission model to simulate traffic congestion on the freeway network and embed a fixed-point algorithm to equilibrate users' mode choice in the long run within the proposed simulation-based optimization framework. Our numerical results reveal that ridesharing incentives have the potential to improve social welfare and reduce congestion. However, we find that providing too many subsidies to ridesharing users may increase congestion levels and thus be counterproductive from a system performance standpoint. We also investigate the impact of transaction fees to a third-party ridesharing platform on social welfare and traffic congestion. We observe that increasing the transaction fee for ridesharing passengers may help in mitigating congestion effects while improving social welfare in the system.

\section{Introduction}

One of the major factors behind road traffic congestion is the low occupancy rates of vehicles which utilize a high amount of road capacity per passenger travelling. In Australia, more than $69 \%$ of individuals drive to work each day, with a further 5\% travelling as passengers [1]. With urban sprawl, the impact of road congestion on the economy and on society is forecasted to increase significantly in future decades [2]. Further examining travel mode choice in Sydney (see Table 1) reveals that the average occupancy of cars is 1.46 , which is aligned with findings from a decade ago [4]. Yet, the average occupancy of private cars is only 1.1-1.2 for commuters [5], which is much less than the typical 4-seat capacity of cars. This suggests that there is potential to increase vehicle occupancy rates through carpooling and ridesharing initiatives. A study conducted by Wang et al. [6] demonstrated that increasing vehicle occupancy rates may yield significant benefits in travel cost and time savings. Furthermore, incentives for commuters have the potential to reduce congestion. This phenomenon has been documented in field and controlled experiments that provided commuters with monetary incentives to promote off-peak departure times $[7,8]$. Ridesharing allows travellers to share vehicles with those who have similar origins, destinations, and schedules on short notice, which may reduce the net number of vehicles in traffic by increasing the utilization of vehicle capacity. This may lead to significant social and environmental benefits [9]. Compared with more traditional road network optimization approaches, e.g., increasing 
Table 1: Resident travel patterns, Sydney [3].

\begin{tabular}{lcc}
\hline \multicolumn{3}{c}{ Mode share (average weekday) } \\
\hline Car driver & $47.2 \%$ & $2012 / 13$ \\
Car passenger & $21.8 \%$ & $2012 / 13$ \\
Train & $5.4 \%$ & $2012 / 13$ \\
Bus & $6.0 \%$ & $2012 / 13$ \\
Walk only & $17.5 \%$ & $2012 / 13$ \\
\hline
\end{tabular}

capacity or adding public transit services, ridesharing can be a cost-effective way to reduce urban congestion. Furthermore, commuting via ridesharing can be encouraged by providing subsidies $[10,11]$.

In this paper, we explore the impact of subsidizing ridesharing users on vehicle occupancy rates in a daily morning commute context. We focus on a case study based on a major motorway in Sydney, Australia, representative of a freeway network with multiple origin-destination pairs. We only consider ridesharing in the sense of carpooling, i.e., wherein, unlike in ride-sourcing markets, ridesharing drivers also commute to their workplace. We consider that users have the option to choose from different roles including solo driver (SD), ridesharing driver (RSD), or ridesharing passenger (RSP) or use a reserve travel option (RO) that does not contribute to congestion effects on the freeway network. We use a multinomial logit model to capture users' mode choice behavior in the long run wherein mode-based utility functions are calibrated based on real data. We use a link transmission model to capture congestion effects on the freeway network. We then proposed a simulation-based optimization framework to determine how subsidies can be used to improve social welfare and reduce congestion in the long run. Numerical experiments on the proposed case study reveal that increasing the amount of subsidy available to ridesharing drivers and passengers tends to increase social welfare. However, we find that although subsidizing ridesharing users may reduce congestion, increasing the amount of subsidy may also increase congestion levels on the freeway. Hence, this study provides nontrivial insights to quantify the tradeoff between subsidizing strategies, welfare, and system performance in a realistic daily morning commute context.

The rest of the paper is organized as follows. The literature review is discussed in Section 1.1, and our contributions are outlined in Section 1.2. The proposed methodology is introduced in Section 2. The data used to create the case study are presented in Section 3. Numerical experiments are reported in Section 4, and concluding remarks are discussed in Section 5.

1.1. Literature Review. Ridesharing has the potential to improve social welfare and has been the topic of several studies in the transportation literature [12-14]. By pooling rides, ridesharing provides opportunities to develop policies for parking pricing discounts, toll pricing, and high occupancy vehicle (HOV) lanes accessibility $[15,16]$. The research of Ma and Zhang [17] shows that controlling parking charge and ridesharing price in certain level may reduce the total travel time and travel cost. However, many workers received free parking place, so the method of parking price control is not suitable for every traveller. Willson and Shoup [18] proposed subsidies and penalties to manage parking fees and incentivize ridesharing. Yet, the application of the proposed mechanism may not be practical from an employers' standpoint, especially for solo drivers. Liu and $\mathrm{Li}$ [19] focus on examining the relationship between system optimum pricing of road tolls and ridership with ridesharing. Their study is thus limited to tolled roads. Furthermore, there exists skepticism around the effectiveness of HOV lanes in the transport community [20, 21]. A recent report indicates that even though the number of HOV lanes has increased in the US, the number of carpooling commuters is declining and the enforcement of the HOV lanes remains a challenge [22]. This suggests that further research is needed to efficiently deploy incentives for promoting ridesharing.

Most existing studies on ridesharing focus on matching algorithms and aim to maximize the profits of the ridesharing operator rather than increasing social welfare or reducing congestion. Simonetto et al. [23] and Gambella et al. [24] develop a dynamic ridesharing algorithm and system which shortens the running time at scale. Pandey et al. [25] present a method to alleviate the service degradation caused by ridesharing company competition. Agatz et al. [26] aim at minimizing the users' travel distance and travel cost and their individual travel costs while Ghoseiri [27]; Wang, Winter, and Tomko [28]; Wang, Agatz, and Erera [29]; and Najmi et al. [30] also aim to find an optimal matching amongst ridesharing drivers and passengers. $\mathrm{Di}$ Febbraro et al. [31] use a discrete event system-based simulation to examine the performance of a ridesharing matching system. He et al. [32] and Aydin et al. [33] attempt to improve ridesharing matching algorithms which consider route choice. Route planning and optimization for ridesharing rides have also received growing attention in the literature [34-36].

Statistical analyses showed that ridesharing could help in reducing congestion and increasing social and environmental benefits [37]. Yet, only a few studies have examined the impact of ridesharing on congestion with the goal to improve social welfare. Xu, Ordóñez, and Dessouky [38] and Xu, Pang, Ordóñez, and Dessouky [39] examined the role of ridesharing in the context of a static traffic equilibrium and explored the impact of fare structures on ridership. However, they do not optimize social welfare in their formulations. Furthermore, static network models have well-known limitations including the lack of queue spillback and wave propagation phenomena. Several studies have used dynamic traffic models within simulation-based optimization frameworks to increase the degree of accuracy of traffic models so as to better inform decision-makers. In particular, network pricing problems have been frequently solved using simulation-based optimization methods [40-44].

In summary, most studies on ridesharing either focus on matching algorithms or examine the impact of ridesharing on congestion using static network models. While several studies have proposed incentive methods to reduce 
congestion, subsidies have not received as much attention as alternative approaches.

1.2. Our Contributions. We make the following contributions to the field. We consider a morning commute problem on a freeway network with multiple origins and destinations. We assume that users have the possibility to choose amongst different roles including SD, RSD, and RSP or use an alternative travel mode, $\mathrm{RO}$, that does not contribute to congestion effects on the freeway. We assume that users aim to maximize their utility and propose utility functions to represent their preferences. We use a logit model to capture users' utilities in the long run and use a dynamic network model to account for the impact of vehicle occupancy rates on congestion effects. We then propose a simulation-based optimization framework to determine optimal subsidies for ridesharing users so as to maximize social welfare.

To illustrate the behavior of the proposed approach, we design a case study based on real data representative of the morning commute on Sydney's M4 Motorway network in Australia. The road network, travellers' demographics data, and travel demand data are derived from open data sources. Our study may help in quantifying the effects of subsidizing ridesharing roles onto social welfare and network congestion, thus providing managerial insights for mobility service providers and transport authorities.

\section{Methodology}

The goal of this study is to investigate the potential of subsidies to increase the ridership of ridesharing travel modes, i.e., ridesharing driver and ridesharing passenger, and reduce congestion so as to improve social welfare. We consider four travel modes, namely, SD, RSD, RSP, and RO, which does not contribute to congestion effects. We first present the mathematical formulation of the utility functions of each travel mode in Section 2.1 before introducing the dynamic network model and the simulation-based optimization framework in Section 2.2.

Table 2 summarizes the notations used throughout the paper.

2.1. Utility Functions and Choice Model. We seek to derive utility functions that are able to capture the preferences of travellers towards the four travel modes considered. The factors which can affect travel demand include transport options, prices, demand management, commercial activity, land use, and demographics [45]. Although social factors, such as the fear of sharing a ride with a potential stranger, could impact the adoption of ridesharing roles, a reliable reputation system could help travellers establish trust with each other [9]. Therefore, we focus on commercial factors including travel cost, parking cost, travel time, and walking time as the main factors which impact the demand for ridesharing.

Let $W$ be the set of OD pairs in the freeway network. The total number of travellers of each OD pair is assumed to be known and constant. Let $Q^{w}$ be the total demand for OD $w \in W$. The travel demand of each OD pair is assumed to be split across a set of travel modes. We denote by $M$ the set of travel modes and consider four modes including solo driver (SD), ridesharing driver (RSD), ridesharing passenger (RSP), and a reserve travel option (RO), denoted by $s, d$, $p$, and $r$, respectively, i.e., $M \equiv\{s, d, p, r\}$. For each mode $m \in M$, we denote by $q_{m}^{w}$ the variable demand for mode $m$ on OD $w \in W, \mathbf{q}=\left[q_{m}^{w}\right]^{|M||W|}=\left[q_{s}^{w}, q_{d}^{w}, q_{p}^{w}, q_{r}^{w}\right]^{|W|}$. The following relationship holds for each OD pair of the network:

$$
Q^{w}=\sum_{m \in M} q_{m}^{w}=q_{s}^{w}+q_{d}^{w}+q_{p}^{w}+q_{r}^{w}, \quad \forall w \in W
$$

Let $P_{m}^{w} \geq 0$ be the proportion of travellers of OD $w \in W$ using mode $m \in M$. The number of users commuting on OD $w$ in mode $m$ is determined as

$$
q_{m}^{w}=Q^{w} P_{m}^{w}, \quad \forall w \in W, \forall m \in M
$$

We assume that the form of utility functions for all travel modes is known. Let $\beta_{m}^{w}$ be the constant part of the utility function of mode $m \in M$ for OD $w \in W$. Let $b_{m}$ be the number of variable terms in the utility functions of mode $m \in M$. Let $\beta_{m}^{w}$ be the constant part of the utility function of mode $m \in M$ for OD $w \in W$. Let $b_{m}$ be the number of variable terms in the utility functions of mode $m \in M$. Let $X_{v, m}^{w}$ be the $v$-th variable in the utility function of mode $m$ for OD $w$, and let $\beta_{v, m}$ be its associated coefficient in the utility function. The utility function for mode $m \in M$ in OD $w \in W$ is

$$
U_{m}^{w} \equiv \beta_{m}^{w}+\sum_{v=1}^{b_{m}} \beta_{v, m} X_{v, m}^{w}, \quad \forall w \in W, \forall m \in M .
$$

We use a multinomial logit model to determine travellers' mode choice proportions [46]. Since the model choice is implicit in our numerical experiments, the mean of the potential error term is zero and the scale parameter is normalized to one. We assume that users' travel mode decisions in the long run follow a logistic distribution; hence, the proportion of users for OD $w \in W$ using mode $m \in M$ is

$$
P_{m}^{w}=\frac{e^{U_{m}^{w}}}{\sum_{n \in M} e^{U_{n}^{w}}}, \quad \forall w \in W, \forall m \in M .
$$

Let $y_{d}^{w} \geq 0$ and $y_{p}^{w} \geq 0$ be the subsidy per unit distance (e.g., in AUD $/ \mathrm{km}$ ) provided to RSD and RSP on OD $w \in W$, respectively. Let $\mathbf{y}=\left[y_{d}^{w}, y_{p}^{w}\right]^{2|W|}$ be the vector of RSD and RSP subsidies. The vector of subsidies $\mathbf{y}$ is the main decision variable of the optimization problem proposed in Section 2.2. We consider that demand for each travel mode is endogenous to mode-based travel times. Since subsidies to RSP are assumed to affect the utility of this travel mode, in the long run, the utilities of other travel modes are also function of $\mathbf{y}$ and so is the travel demand across all modes and OD pairs. To capture these phenomena, we denote by $\mathbf{q}(\mathbf{y})$ the travel demand in the network and write OD- and modebased utilities as functions of this variable demand vector.

Let $T T_{m}^{w}(\mathbf{q}(\mathbf{y}))$ be the travel time of OD $w \in W$ and mode $m \in M$. This travel time is a function of the demand $\mathbf{q}(\mathbf{y})$, which is itself function of the subsidy vector $\mathbf{y}$. Let 
TABLE 2: Signs and notations.

\begin{tabular}{|c|c|}
\hline Notation & Interpretation \\
\hline $\begin{array}{l}\beta_{v, m} \\
P m\end{array}$ & Regression coefficient associated with the explanatory variable $v$ and in mode $m$ \\
\hline$P_{w}^{m}$ & Probability of choosing mode $m$, OD $w$ \\
\hline $\mathbf{q}$ & Demand matrix \\
\hline$Q^{w}$ & Demand of OD $w$ \\
\hline$q_{w}^{m}$ & Travellers of choosing mode $m$, OD $w$ \\
\hline$U_{w}^{m}$ & Utility value of travellers of mode $m$, OD $w$ \\
\hline$X_{v, m}$ & Regression variable associated with the explanatory variable $v$ and in mode $m$ \\
\hline $\mathbf{y}$ & Subsidy matrix \\
\hline$y^{w}$ & Subsidy to OD $w$ \\
\hline$c$ & Capacity (available seats) of ridesharing vehicles \\
\hline$d$ & Ridesharing driver \\
\hline$p$ & Ridesharing passenger \\
\hline$r$ & Reserve options (e.g., train or bus) \\
\hline$s$ & Solo driver \\
\hline$M$ & Travel modes include $d, p, r$, and $s$ \\
\hline$m$ & Mode $m$ in $M$ \\
\hline$W$ & OD matrix \\
\hline $\mathbf{w}$ & OD pair in $W$ \\
\hline FC & Financial cost for holding a vehicle per day \\
\hline$E C^{w}$ & Energy cost for OD $w$ \\
\hline$T C_{m}^{w}$ & Travel cost of mode $m$, OD $w$ \\
\hline$B C$ & Basic cost of ridesharing passenger \\
\hline$D^{w}$ & Distance of OD $w$ \\
\hline$T T_{m}^{w}$ & Travel time of mode $m$, OD $w$ \\
\hline$r_{T T}$ & Payment rate of travel time \\
\hline$r_{D}$ & Payment rate of travel distance \\
\hline$r_{B}$ & Fraction of ridesharing passenger payment for booking ride \\
\hline$C S$ & Consumer surplus \\
\hline$I$ & Total subsidy investment \\
\hline$T$ & Total transaction fees \\
\hline SW & Social welfare of the transport system \\
\hline $\bar{y}$ & The upper bound of the subsidy per unit distance \\
\hline
\end{tabular}

$T C_{m}^{w}(\mathbf{y})$, assumed to be a function of the demand, and $W T_{m}^{w}$ be the travel cost and the walking time for OD $w \in W$ and mode $m \in M$, respectively. Let $P C^{w}$ be the parking cost for OD $w \in W$. For each mode $m \in M$, we denote by $\beta_{T C, m}$ the travel cost coefficient, we denote by $\beta_{W T, m}$ the walking time coefficient, we denote by $\beta_{T T, m}$ the travel time coefficient, and we denote by $\beta_{P C, m}$ the parking cost coefficient.

The utility for the RSD mode on OD $w \in W$ is

$$
\begin{aligned}
U_{s}^{w}(\mathbf{y})= & \beta_{s}^{w}+\beta_{T C, s} T C_{s}^{w}(\mathbf{y})+\beta_{W T, s} W T_{s}^{w}+\beta_{T T, s} T T_{s}^{w}(\mathbf{q}(\mathbf{y})) \\
& +\beta_{P C, s} P C^{w} .
\end{aligned}
$$

The utility for the RSD mode on OD $w \in W$ is

$$
\begin{aligned}
U_{d}^{w}(\mathbf{y})= & \beta_{d}^{w}+\beta_{T C, d}\left(T C_{d}^{w}(\mathbf{y})-y_{d}^{w}\right)+\beta_{W T, d} W T_{d}^{w} \\
& +\beta_{T T, d} T T_{d}^{w}(\mathbf{q}(\mathbf{y}))+\beta_{P C, d} P C^{w} .
\end{aligned}
$$

The utility of the RSP mode on OD $w \in W$ is

$$
\begin{aligned}
U_{p}^{w}(\mathbf{y})= & \beta_{p}^{w}+\beta_{T C, p}\left(T C_{p}^{w}(\mathbf{y})-y_{p}^{w}\right)+\beta_{W T, p} W T_{p}^{w} \\
& +\beta_{T T, p} T T_{p}^{w}(\mathbf{q}(\mathbf{y})) .
\end{aligned}
$$

The utility of the RO mode on OD $w \in W$ is

$$
U_{r}^{w}=\beta_{r}^{w}+\beta_{T C, r} T C_{r}^{w}+\beta_{W T, r} W T_{r}^{w}+\beta_{T T, r} T T_{r}^{w} .
$$

In this study, we assume that the $\mathrm{RO}$ is not influenced by congestion; hence the utility of the $\mathrm{RO}$ is assumed to be a constant. The utility parameters of each mode are determined using an initial traffic simulation. This is used to match the baseline mode shares in Table 3. Details on this calibration procedure are provided in section 4.1.

Vehicle ownership cost may include various vehicle related costs, e.g., depreciation, financing, insurance, registration, and repairs, which are assumed fixed and uniform across OD pairs [47]. We denote such fixed costs by FC. Energy costs for OD $w \in W$ are assumed to be function of the OD travel time and thus of mode-based demand and are denoted by $E C^{w}(\mathbf{q}(\mathbf{y}))$. Hence, the travel cost function of $\mathrm{SD}$ is

$$
T C_{s}^{w}(\mathbf{y}) \equiv F C+E C^{w}(\mathbf{q}(\mathbf{y})), \quad \forall w \in W .
$$

This cost should be borne by vehicles owners including SD and RSD. However, RSD are expected to decrease their travel cost by receiving payments from RSP. We consider that RSP pay RSD based on trip travel time and trip distance. Let $D^{w}$ be the trip distance of OD $w \in W$ and let $r_{D}$ be the valuation of travel distance. Furthermore, let $r_{T T}$ be the valuation of travel time and let $B C$ be the base cost for using the ridesharing system. We assume that a fraction of 
TABLE 3: Initial mode split.

\begin{tabular}{lc}
\hline Mode & Proportion (\%) \\
\hline Solo driver & 34.33 \\
Ridesharing driver & 22.88 \\
Ridesharing passenger & 26.42 \\
Reserve options & 16.36 \\
\hline
\end{tabular}

passengers' travel cost denoted by $r_{B}$ is allocated to the ridesharing booking platform.

We assume that RSP cannot earn revenue by using ridesharing services even with a subsidy. Accordingly, we define the travel cost of RSP as the maximum between the difference of presubsidy travel cost including booking fees and the trip subsidy and zero. We assume that presubsidy travel cost for RSP is $B C+r_{D} D^{w}+r_{T T} T T_{p}^{w}(\mathbf{q}(\mathbf{y}))$ and this cost is augmented by dividing it by $\left(1-r_{B}\right)$ to account for booking fees. The trip subsidy is determined as the per-unit RSP subsidy times the trip distance, i.e., $y_{p}^{w} D^{w}$. Accordingly, the RSP travel cost is defined as

$$
T C_{p}^{w}(\mathbf{y}) \equiv \max \left\{\frac{B C+r_{D} D^{w}+r_{T T} T T_{p}^{w}(\mathbf{q}(\mathbf{y}))}{1-r_{B}}-y_{p}^{w} D^{w}, 0\right\},
$$

$\forall w \in W$

Unlike RSP, we assume that RSD may earn revenue from providing ridesharing services. To determine the travel cost of RSD, we first deduct from equation (9) the trip subsidy, i.e., $y_{d}^{w} D^{w}$, and similarly to the RSP take the maximum between this difference and zero. This aims to represent the fact that RSD travel cost cannot be oversubsidized. We then deduct the total payments from RSP from this amount. The average total payment from RSP can be determined by multiplying the numerator within equation (10), which represents RSP travel cost before subsidy and booking fees, by the ratio of RSP to RSD users on each OD pair. Accordingly, the travel cost of RSD is

$$
\begin{aligned}
T C_{d}^{w}(\mathbf{y})= & \max \left\{T C_{s}^{w}(\mathbf{y})-y_{d}^{w} D^{w}, 0\right\}-\left(1-r_{B}\right) \frac{q_{p}^{w}}{q_{d}^{w}} \\
& \cdot\left(B C+r_{D} D^{w}+r_{T T} T T_{p}^{w}(\mathbf{q}(\mathbf{y}))\right), \quad \forall w \in W .
\end{aligned}
$$

The term $\max \left\{T C_{s}^{w}(\mathbf{y})-y_{d}^{w} D^{w}, 0\right\}$, in (11), is the travel cost after subsidy. To prevent RSD from being oversubsidized, we impose that the subsidy cannot exceed the cost of SD, i.e., the fixed cost of vehicles and energy cost of travel. The term $\left(1-r_{B}\right)\left(q_{p}^{w} / q_{d}^{w}\right)\left(B C+r_{D} D^{w}+\right.$ $\left.r_{T T} T T_{p}^{w}(\mathbf{q}(\mathbf{y}))\right)$ describes the income from RSP which depends on the travel cost of RSP after transaction and the number of passengers per vehicle.

We use the logsum measure to capture the change in the expected consumer surplus. Let 0 be a null vector of appropriate size corresponding to the no-subsidy case, i.e., the baseline configuration. Let $\alpha$ be the marginal utility of travel cost. We denote by $C S(\mathbf{y})$ the change in the expected consumer surplus relative to the baseline configuration where no subsidies are available. This change can be calculated as

$$
C S(\mathbf{y})=\frac{1}{\alpha} \sum_{w \in W} Q^{w}\left[\ln \left(\sum_{m \in M} e^{U_{m}^{w}(\mathbf{y})}\right)-\ln \left(\sum_{m \in M} e^{U_{m}^{w}(0)}\right)\right] .
$$

CS (y) represents the total difference of custom surplus in the system between after and before applying subsidies. Let $T(\mathbf{y})$ be the total transaction fee charged to ridesharing passengers by the service platform, i.e., $T(\mathbf{y})=\sum_{w \in W} r_{B} T C_{p}^{w}(\mathbf{y})$. Let $I(\mathbf{y})$ be the total subsidy investment, i.e., $I(\mathbf{y})=\sum_{w \in W} \sum_{m \in M} y_{m}^{w}$. The social welfare of the system, denoted by $S W(\mathbf{y})$, is

$$
S W(\mathbf{y})=C S(\mathbf{y})-I(\mathbf{y})-T(\mathbf{y}) .
$$

The goal of the proposed approach is to maximize social welfare by optimizing the subsidy vector $\mathbf{y}$ while accounting for traffic congestion and mode choice effects. We next introduce the proposed simulation-based optimization approach.

2.2. Simulation-Based Optimization Approach. To optimize social welfare while accounting for congestion effects induced by varying ridesharing subsidies and their impact on users' mode choice, we introduce a simulation-based optimization approach built on an iterative equilibrium model.

To model traffic dynamics and its impact on congestion, we use the Link Transmission Model (LTM), a numerical solution method for dynamic network loading [48]. The LTM simulates traffic on the transport network under fixed demand. To account for the effect of ridesharing subsidies onto users' mode choice we use a classical fixed-point algorithm that iteratively simulates traffic to obtain OD- and mode-based travel times and updates utility functions (5), (6), and (7) accordingly. We use the Method of Successive Averages (MSA) to iteratively equilibrate mode split proportions for each OD based on OD- and mode-based travel times. The iterative procedure which consists of alternating between traffic simulation using the LTM and equilibrating OD- and mode-based demand $\mathbf{q}(\mathbf{y})$ for a given subsidy vector $\mathbf{y}$ is hereby referred to as simulation $(\mathbf{y})$.

Let $q_{m}^{w, n-1}$ be the demand of mode $m \in M$ for OD $w \in W$ at the $n-1$-th iteration of procedure simulation $(\mathbf{y})$. Let $q_{m}^{w, \star}$ be demand of mode $m \in M$ for OD $w \in W$ obtained after simulating traffic using the LTM and determining mode choice proportion using the logit model (4). The demand at the $n$-th iteration is determined as

$$
q_{m}^{w, n}=\frac{1}{n} q_{m}^{w, \star}+\frac{n-1}{n} q_{m}^{w, n-1} \quad \forall w \in W, \forall n \in N .
$$

Convergence of the procedure simulation $(y)$ is assumed to be achieved when the OD- and mode-based demand vector $\mathbf{q}(\mathbf{y})$ remains near-constant after two consecutive iterations. Specifically, let $\delta^{n}$ be the total absolute difference between iterations $n-1$ and $n$ : 


$$
\delta^{n}=\sum_{w \in W} \sum_{m \in\{s, d\}}\left|q_{m}^{w, n}-q_{m}^{w, n-1}\right|
$$

We consider that procedure simulation(y) has converged if $\delta^{n} \leq \varepsilon_{q}$ for a predetermined $\varepsilon_{q}$ value.

To formulate the proposed ridesharing subsidy optimization problem, we embed the procedure simulation $(\mathbf{y})$ as a constraint of the simulation-based optimization formulation and assume that the output of simulation $(\mathbf{y})$ gives an equilibrium demand vector $\mathbf{q}(\mathbf{y})$ which affects the utility functions of the travel modes considered. In addition to utility equations, we consider two constraints in the proposed optimization formulation. We assume that all per-unit subsidies are upper-bounded by the maximum available perunit subsidy $\bar{y}$. Furthermore, we assume that the average occupancy rate is upper-bounded by the vehicle capacity $c$.

The resulting simulation-based optimization approach is summarized in equations (16a)-(16d):

maximize $S W(\mathbf{y})$,

subject to

utility functions : (5), (6), (7), $\forall w \in W$,

$$
\begin{aligned}
& 0 \leq y_{m}^{w} \leq \bar{y}, \forall w \in W, \forall m \in\{d, p\} \\
& \frac{q_{d}^{w}+q_{p}^{w}}{q_{d}^{w}} \leq c, \forall w \in W, \\
& \mathbf{q}(\mathbf{y})=\operatorname{simulation}(\mathbf{y}) .
\end{aligned}
$$

Equation (16a) is the objective function which aims to maximize social welfare. Constraint (16b) sets bounds on subsidy variables. Constraint (16c) is the capacity constraint. Constraint (16d) imposes that the demand vector $\mathbf{q}(\mathbf{y})$ is an equilibrated, logit-compatible split of the travel demand across ODs and modes which accounts for congestion effects.

To solve the proposed simulation-based optimization formulation (16a)-(16d) we use a heuristic algorithm from an off-the-shelf global optimization toolbox which aims to optimize the subsidy vector $y$ until the total absolute value of the difference of two consecutive iterations is less than a predetermined threshold $\varepsilon_{y}$. The flowchart of the proposed simulation-based optimization framework is illustrated in Figure 1.

\section{Data}

In this section, we present the data used in this study.

3.1. Freeway Network. We consider a freeway network based on the M4 Motorway in Sydney, Australia, with multiple OD pairs. The traffic demand is obtained based on census data from regions nearby the freeway [49]. The M4 Motorway is an important road which connects Western Sydney to Sydney's Central Business District (CBD). The M4
Motorway serves more than 40,000 trips per day in a single direction [50]. Due to congestion levels, the average speed along the M4 Motorway is about 50\% less than the maximum speed limit during peak hours [51].

The morning commute (from West to CBD) is particularly critical from a congestion standpoint, based on trips departure time [52] and compared with the traffic volumes on the Great Western Highway [53]. The number of travellers using motorised modes peaks at around 8:30 AM. The M4 Motorway is tolled on some parts of the road (WestConnex) [54]. In our study, we focus on the nontolled part of the motorway which covers over $70 \%$ of its length. As noted by Terrill and Institute [1] the traffic flow towards the CBD is significantly higher than that from the CBD; hence we focus on Eastbound traffic only in our study. The portion of the M4 Motorway considered in this study is depicted in Figure 2. The transport network was obtained from OpenStreetMap [55]. The capacity of the motorway is set to 2100 vehicle/h/ lane, the free-flow speed is $100 \mathrm{~km} / \mathrm{h}$, and the jam density is 180 vehicle $/ \mathrm{km} /$ lane $[56,57]$. The road between B1 and A48 has three lanes and the rest of the freeway network considered has four lanes.

3.2. Travel Demand Data. We focus on the Eastbound morning commute between 6:30 am and 9:30 am. The numbers of travellers travelling to work are taken from Australian Census [58]. Those who usually live nearby the M4 Motorway and have workplaces to the East of the usual residences are the potential travellers taking vehicles on M4 Motorway. Between 6:30 and 9:30 am, there are 60\% trips belonging to nondiscretionary [4] and they are considered as the commuters represented in the census data. To determine an initial travel mode split between SD and RSD (which is not provided in the census data), we make the following assumption. Since $60 \%$ of the trips belong to nondiscretionary between 6:30 and 9:30 am [4] and the commuting occupancy is close to 1 , we assume that $60 \%$ of the vehicle drivers are SD and the other drivers are RSD. The resulting baseline travel mode split is given in Table 1 .

To determine the travel demand we make the following assumptions. According to Traffic Volume Viewer [50], the flow on the M4 is around $60 \%$ of the sum of the M4 Motorway and the Great Western Highway which is a substitute for M4 Motorway. The vehicle flow from point B1 is $3056.25 \mathrm{vph}$ in morning peak, while it is 34964 vehicles in one weekday (see Figure 2). This corresponds to $8.7 \%$ of the total flow. Note that there are no off-ramps at the B2, B4, B7, and $\mathrm{B} 8$ sites. From the above information and census data, we obtain the hourly travel demand matrix summarized in Table 4.

The traffic flows from $\mathrm{B} 1$ in Table 4 (top row) are regarded as fixed background flows which are not considered in the optimization and are assumed fixed. The coefficients of the logit model are shown in Table 5 and there is only a constant value for $\mathrm{SD}\left(\beta_{s}=0.575\right)$, which is the mean of other parameters from Ciari and Axhausen [10]. The unit for cost is Australian dollars (AUD) and the unit for time is 


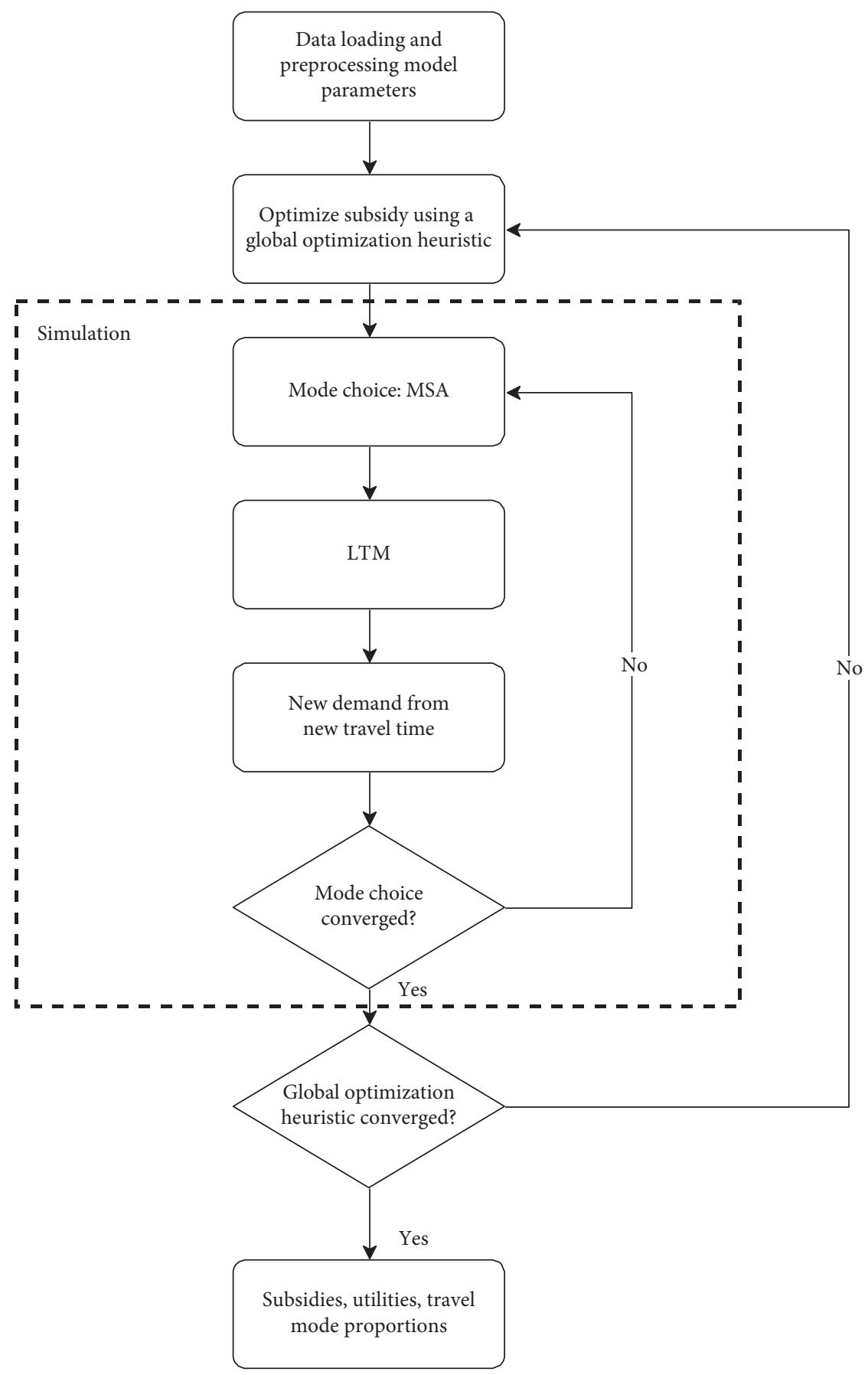

FIGURE 1: Flowchart of the simulation-based optimization framework. The procedure simulation(y) is shown with a dashed line.

minutes. For other modes, the constant coefficient values are determined as discussed in Section 2.

We assume that the value of time is $13.73 \mathrm{AUD} / \mathrm{h}$ for every mode $[59,60]$, which is more conservative than values used in the research of Hensher et al. [61]. According to Schuster et al. [47], the financial cost of a vehicle ownership is 6150.5 USD per year and the cost rate to RSP is selected as $3.9 \mathrm{USD} / \mathrm{h}, 0.39 \mathrm{USD} / \mathrm{mile}$, and 10USD monthly cost. We convert USD values to AUD by assuming a $50 \%$ increase. The number of trips of vehicle owners is 2 trips per day and the usage of RSP is $26.42 \%$ in a workday. Accordingly, the constants in equation (10) are set to $B C=2.84 \mathrm{AUD}$, $r_{T T}=3.73 \mathrm{AUD} / \mathrm{h}$, and $r_{D}=0.41 \mathrm{AUD} / \mathrm{km}$. In equation (9) FC is set to 12.64 AUD per trip. Since travel times are expected to be less than a half hour, the impact of travel time on energy cost is assumed negligible and the fuel cost is set to $0.12 \mathrm{AUD} / \mathrm{km}$.

\section{Numerical Experiments}

In this section, we present the numerical experiments conducted and discuss numerical results. 


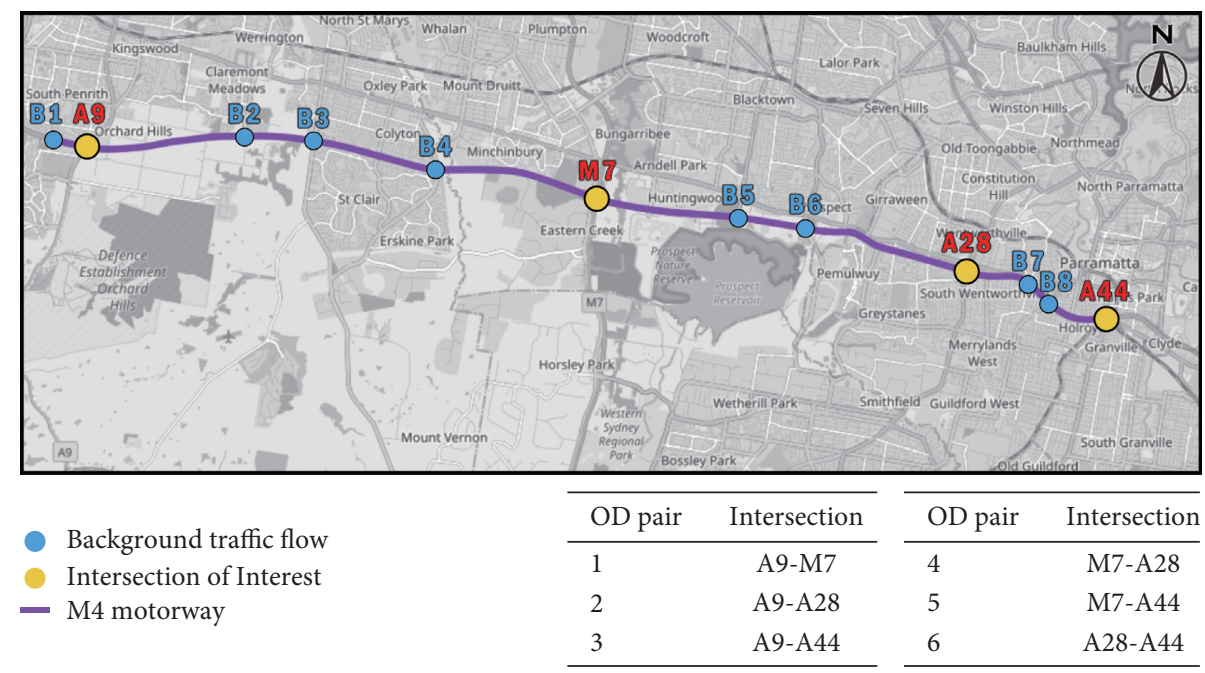

FIgure 2: Sydney's M4 Motorway.

TABLe 4: Total demand.

\begin{tabular}{|c|c|c|c|c|c|c|}
\hline & B3 & M7 & B5 & B6 & A28 & A44 \\
\hline B1 & 445.65 & 551.42 & 157.24 & 193.15 & 592.15 & 1116.64 \\
\hline A9 & 310.06 & 382.90 & 109.18 & 134.12 & 411.19 & 775.38 \\
\hline B2 & 247.56 & 306.26 & 87.44 & 107.21 & 328.92 & 620.28 \\
\hline B3 & & 232.81 & 82.88 & 106.60 & 286.19 & 508.66 \\
\hline B4 & & 348.38 & 139.75 & 180.81 & 497.10 & 901.14 \\
\hline M7 & & & 175.79 & 258.21 & 709.99 & 1557.15 \\
\hline B5 & & & & 17.49 & 514.44 & 1035.72 \\
\hline B6 & & & & & 716.38 & 1445.38 \\
\hline A 28 & & & & & & 4928.14 \\
\hline B7 & & & & & & 594.12 \\
\hline B8 & & & & & & 594.12 \\
\hline
\end{tabular}

TABLE 5: Utility parameters.

\begin{tabular}{lcc}
\hline Mode & Utility parameters & Value \\
\hline \multirow{2}{*}{ All } & Travel cost & -0.05524 \\
& Walking time & -0.0438 \\
\hline \multirow{2}{*}{ SD } & Constant & 3.4994 \\
& Travel time & -0.03 \\
& Parking cost & -0.06311 \\
\hline \multirow{2}{*}{ RSD } & Constant & - \\
& Travel time & -0.0348 \\
\multirow{2}{*}{ RSP } & Parking cost & -0.149511 \\
& Constant & - \\
RO & Travel time & -0.0379 \\
\hline
\end{tabular}

4.1. Experiments' Design. The utility parameters of each mode are determined using an initial traffic simulation based on the mode split provided in Table 3 . The RO utility $U_{r}^{w}$ is determined from equations (3) and (4) and $U_{s}^{w}$, where the utility of $\mathrm{SD}, U_{s}^{w}$, is obtained from an initial simulation using equation (5). After this initial simulation, the utility of other modes is determined as follows:

$$
U_{a}^{w}=U_{s}^{w} \frac{P_{a}^{w}}{P_{s}^{w}}, \quad \forall w \in W, a \in M \backslash\{s\} .
$$

Similarly, the constant parameters of $U_{d}^{w}$ and $U_{p}^{w}$ are determined after the initial simulation using equations (6) and (7). Furthermore, in our numerical experiments we require that the RSD and RSP subsidies per unit of distance be identical across ODs. That is, $y_{d}^{w}=y_{D}$ and $y_{p}^{w}=y_{p}$ for all $w \in W$. We assume that vehicles are homogeneous and that their capacity is $c=3$. The proposed simulation-based optimization framework (see Figure 1) is implemented on a desktop computer using MATLAB. The LTM toolbox $[62,63]$ is used to solve the LTM numerically. For the procedure simulation $(\mathbf{y})$, we use the stopping criterion $\varepsilon_{q}=0.001 \sum_{w \in W} Q^{w}$, which represents a total deviation between two consecutive iterations of less than $0.1 \%$ of the total demand. MATLAB's Pattern Search procedure is used as the global optimization heuristic to iteratively optimize the subsidy vector $\mathbf{y}$ with a threshold of $\varepsilon_{y}=0.001$, i.e., less than 1 cent.

4.2. Simulation Results. Two types of indicators are used to describe the performance of the transport system and that 


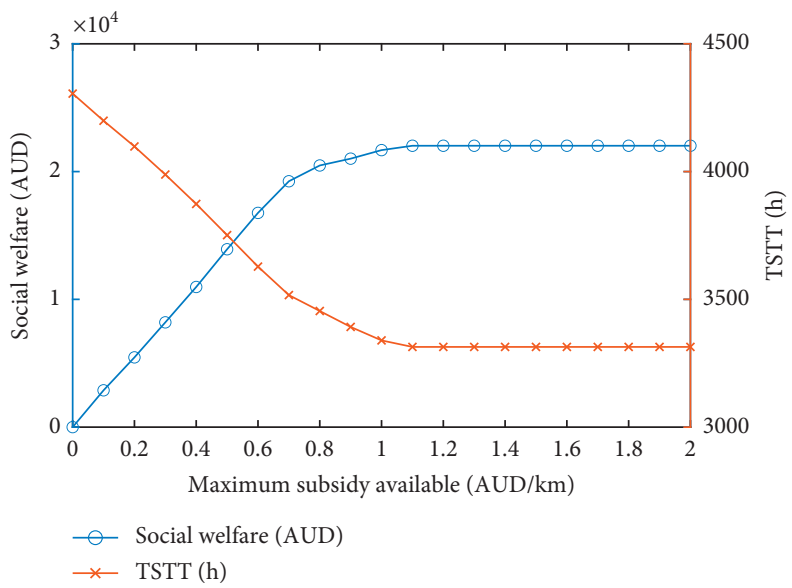

FIgURE 3: Social welfare and TSTT as functions of subsidy.

impact on the OD pairs considered for subsidy allocation. One of the key factors is social welfare which is the expected consumer surplus minus the total subsidy investment. Another indicator of performance is the total system travel time (TSTT) which indicates the congestion level. TSTT is the total travel time of on-road vehicles consisting of SD and RSD.

We examine the behavior of the system via a series of experiments for varying model parameters. We explore the impact of the maximum subsidy available in Section 4.2.1 and the impact of the platform booking (transaction) fee in Section 4.2.2. In the former, we vary the maximum available subsidy from 0 to $2 \mathrm{AUD} / \mathrm{km}$ with a step of $0.1 \mathrm{AUD} / \mathrm{km}$ for a fixed booking fee of $r_{B}=20 \%$. In the latter, we consider six values between 0 and $50 \%$ in steps of $10 \%$ with a maximum subsidy of $\bar{y}=1.5 \mathrm{AUD} / \mathrm{km}$.

4.2.1. Impact of the Maximal Subsidy on Welfare and System Performance. The impact of the maximum subsidy available on social welfare and the TSTT for a fixed transaction rate $r_{B}=20 \%$ is depicted in Figure 3. TSTT decreases while increasing the maximum subsidy available from 0 to 1.3 AUD $/ \mathrm{km}$, and the decrease is faster before $0.7 \mathrm{AUD} / \mathrm{km}$. Then, it slows down before becoming steady at $1.3 \mathrm{AUD} / \mathrm{km}$. The trend of social welfare is reciprocal to that of TSTT for a maximum available subsidy of $1.1 \mathrm{AUD} / \mathrm{km}$. Beyond this value, both social welfare and TSTT plateau.

Figure 4 summarizes the impact of the maximum available subsidy on each of the 6 main ODs in the network. In Figure 4(a), the changes of vehicle occupancy rates of main ODs as a function of the maximum subsidy available are depicted. All of the occupancy rates on the ODs of interest increase until the maximum subsidy available reaches $1.1 \mathrm{AUD} / \mathrm{km}$. We find that the impact on the occupancy rate is more significant for longer ODs, since the trends of A9-A28 and A9-A44 in these two ODs are sharper. Figure 4(b) shows the speed variation of each of the 6 main ODs as a function of the maximum subsidy available. We find that there is almost no impact of subsidy on OD A28A44 because the speed of this OD is near the free-flow speed.
On the other ODs, the speeds increase until the subsidy is $1.1 \mathrm{AUD} / \mathrm{km}$.

Figure 4(c) shows the variation of the RSP travel cost as a function of the maximum subsidy available, while Figure 4(d) shows that of RSD. From Figure 4(c), we observe that the RSP travel cost of the ODs except for A28-A44 decreases with the maximum subsidy available until the subsidy matches the travel cost. However, A28-A44 stops decreasing before zero. Hence, subsidy investment sometimes may not meet the same benefit return. The negative value in Figure 4(d) means that RSD earn revenue from ridesharing services by providing rides to RSP. Except for ODs M7-A28 and A28-A44, RSD are expected to earn revenue. As expected, RSP with shorter travel distance need higher per-unit subsidies to cover their travel cost due to the fixed part of trip costs.

Figure 5 depicts the variation of the allocated subsidies as a function of the maximum subsidy available. It illustrates that the optimal subsidy to RSD is not equal to the maximum available subsidy. This means that allocating the maximum subsidy may not always be the best strategy for every trip mode to improve the social welfare. Furthermore, we observe that beyond $1.1 \mathrm{AUD} / \mathrm{km}$ both subsidies to RSD and RSP plateau.

The elasticities of RSD and RSP for the main OD pairs to subsidy (travel cost reduction) for the calibrated baseline model are reported in Table 6 . Since the initial subsidy is null, we use the midpoint arc elasticity formula [45]. The situation after subsidy is set to $1.1 \mathrm{AUD} / \mathrm{km}$ which is the maximum available subsidy when the scheme is optimal. The mean elasticity of the main ODs and the weighted mean based on the total demand are also indicated in Table 6. It can be seen that RSP is more sensitive to subsidy than RSD and that the elasticity on longer ODs is higher than that on shorter ODs.

4.2.2. Impact of the Transaction Fee Rate on Welfare and System Performance. Figure 6 displays the variation of social welfare and TSTT for a varying transaction fee rate, i.e., ridesharing platform booking fees. Overall, TSTT increases 


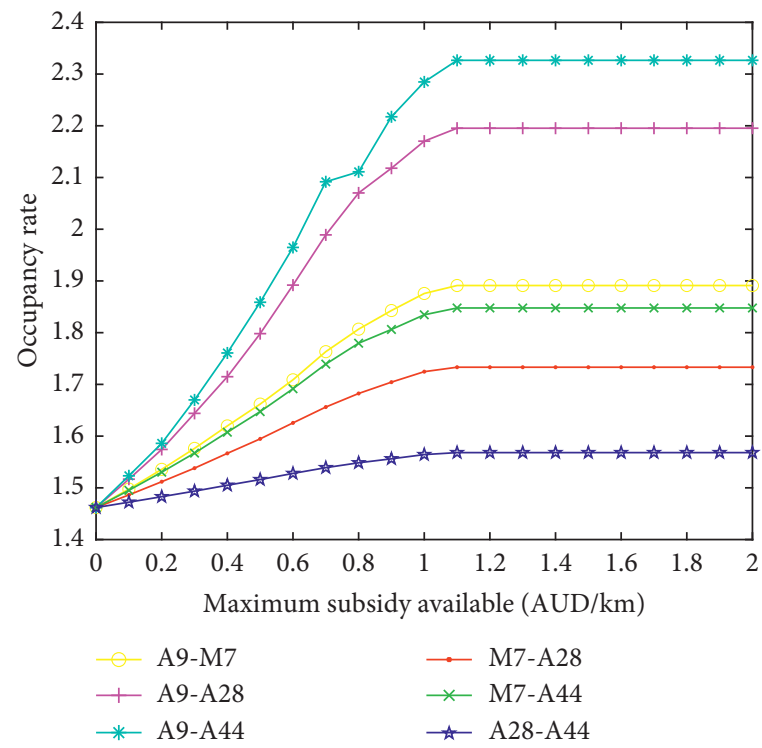

(a)

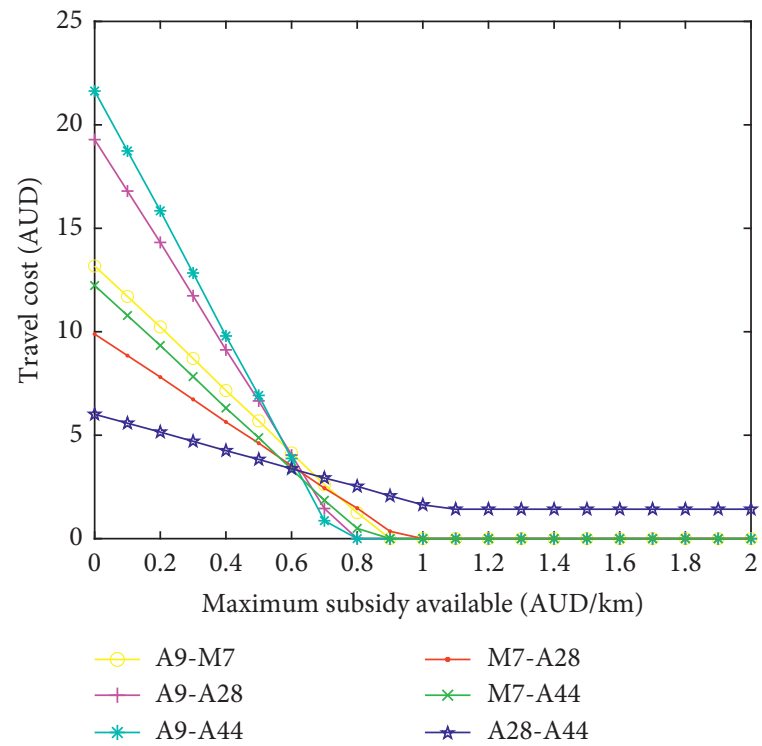

(c)

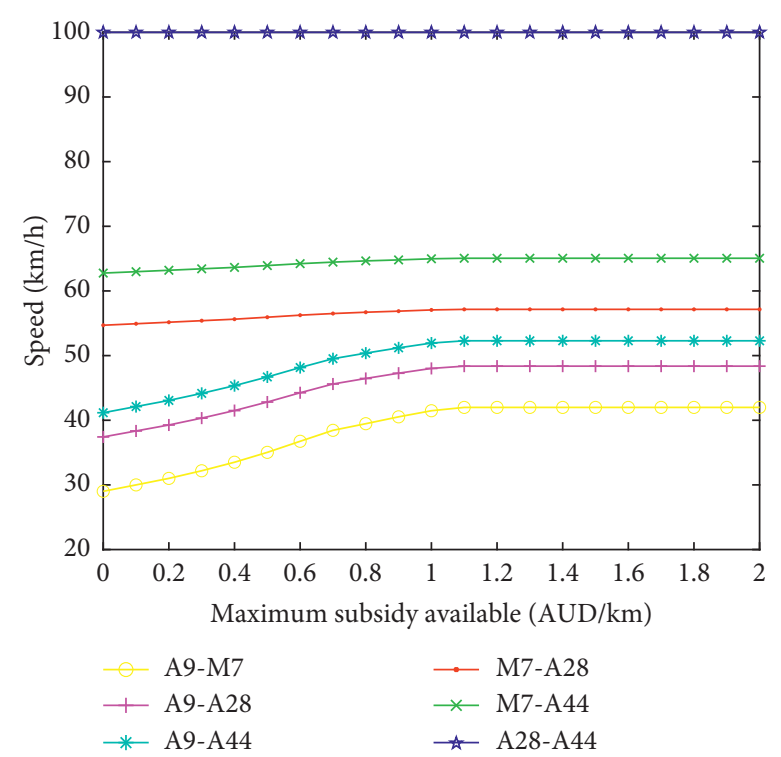

(b)

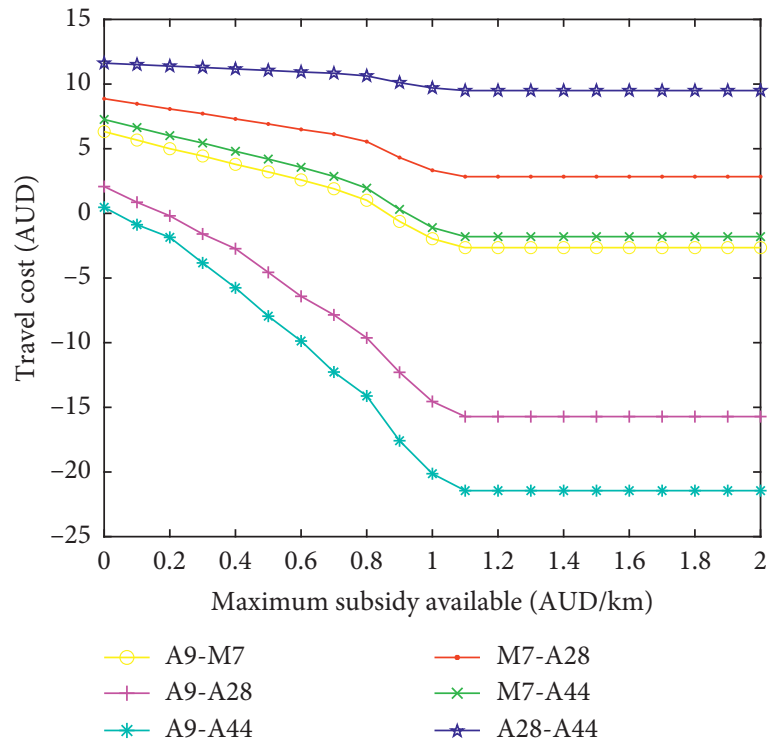

(d)

Figure 4: Detailed analysis of the impact of the maximum subsidy available on the 6 main ODs in the network. (a) Vehicle occupancy. (b) Speed. (c) Average RSP travel cost. (d) Average RSD travel cost.

with the transaction fee rate while social welfare declines. TSTT only slightly reduces between $30 \%$ and $40 \%$ which is possibly due to the heuristic optimization approach used.

The impact of the transaction fee rate on the ODs of interest is summarized in Figure 7. Figure 7(a) shows the trend of the vehicle occupancy rate as a function of the transaction fee rate. We find that the occupancy rates on the main ODs decrease with the transaction fee rate. ODs with a longer distance are more sensitive to the transaction fee rate. Figure 7(b) shows the variation of speed on the main ODs as a function of the transaction fee rate. Except for OD A28A44 which is unaffected by congestion, the trend of average speeds across ODs is aligned with the change of the occupancy rate in Figure 7(a).

Figures $7(\mathrm{c})$ and $7(\mathrm{~d})$ reveal the relationship between travel cost and transaction fee rate for each OD of interest. As expected, the RSP travel cost increases with the transaction fee rate. The travel cost of RSD also increases with the transaction fee rate but at significantly lower growth rate compared to RSP. Figure 8 depicts the trend of the allocated subsidies as a function of the maximum subsidy available. Overall, the allocated subsidy to RSP reduces with the transaction fee rate. This suggests that higher transaction fee rates reduce the benefits from subsidies. 


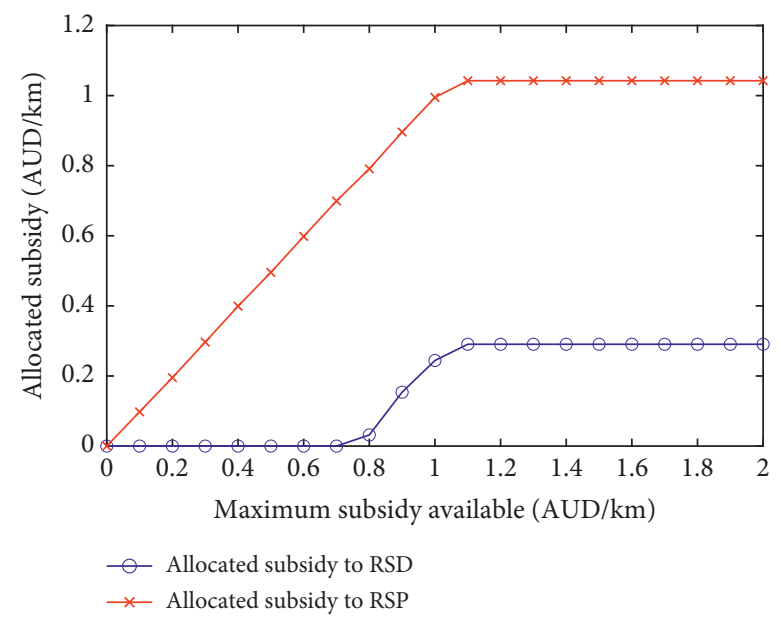

Figure 5: Subsidy allocated as a function of subsidy.

TABle 6: Elasticity of mode demand to subsidy.

\begin{tabular}{lcccccccc}
\hline OD & A9-M7 & A9-A28 & A9-A44 & M7-A28 & M7-A44 & A28-A44 & Mean & $W$-mean \\
\hline RSD & 0.047 & 0.076 & 0.081 & 0.027 & 0.040 & 0.007 & 0.047 & 0.026 \\
RSP & 0.237 & 0.317 & 0.342 & 0.167 & 0.214 & 0.077 & 0.226 & 0.151 \\
\hline
\end{tabular}

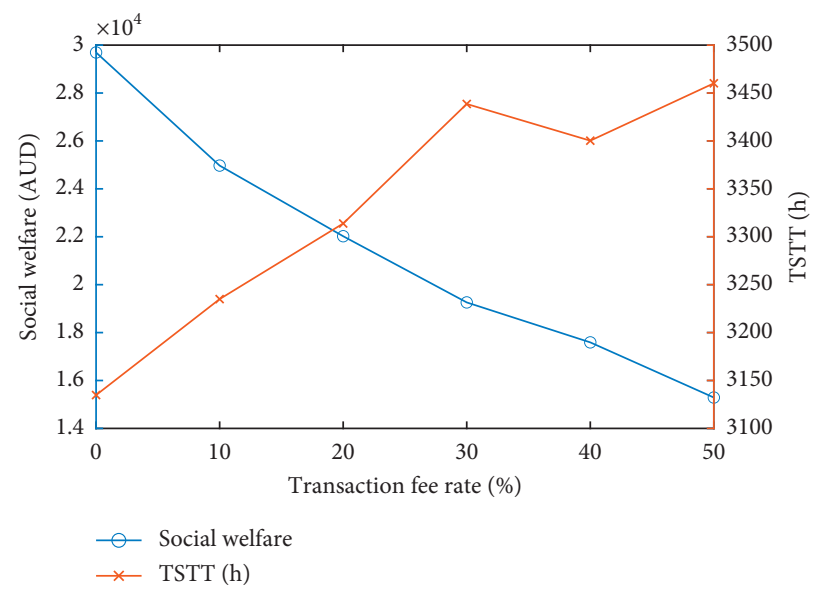

Figure 6: Social welfare and TSTT as functions of transaction fee rate.

\section{Discussion}

Throughout our numerical experiments, we find that subsidizing ridesharing users can increase social welfare. This highlights the tradeoffs between the proportion of ridesharing users and the expected change in consumer surplus as a result of deploying subsidies. Our results also reveal that increasing the available subsidy to ridesharing users may be superfluous, thus providing managerial insights for travel demand management. The plots in Figures 4(c) and 4(d) show that the travel costs of ridesharing users on the main six ODs stabilize after a certain subsidy which is aligned with the vehicle occupancy rate observed on these ODs beyond this threshold. Furthermore, the maximum available subsidy is not always the optimal one. As shown in Figures 5 and 8, the subsidy to RSD is always below the maximum available subsidy. The impact of the transaction fee rate shows that social welfare decreases and the average vehicle occupancy rate tends to decrease with the transaction fee rate. In turn, TSTT increases with the transaction fee rate. RSD and RSP subsidies tend to decrease with the transaction fee rate. This highlights the tradeoffs between consumer surplus and subsidy investment. This suggests that policies supporting a relatively high transaction fee rate may not be beneficial from a system perspective. 

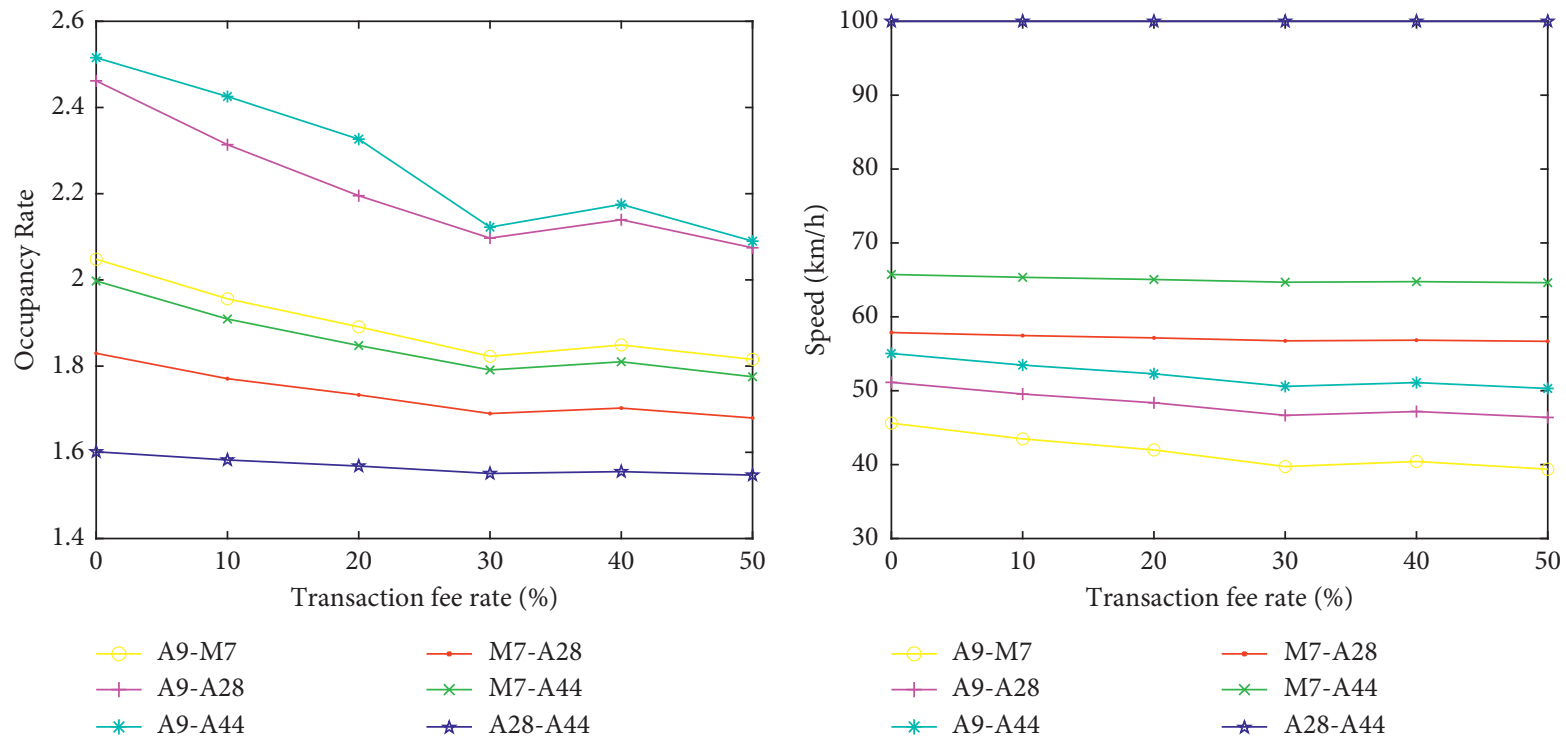

(b)
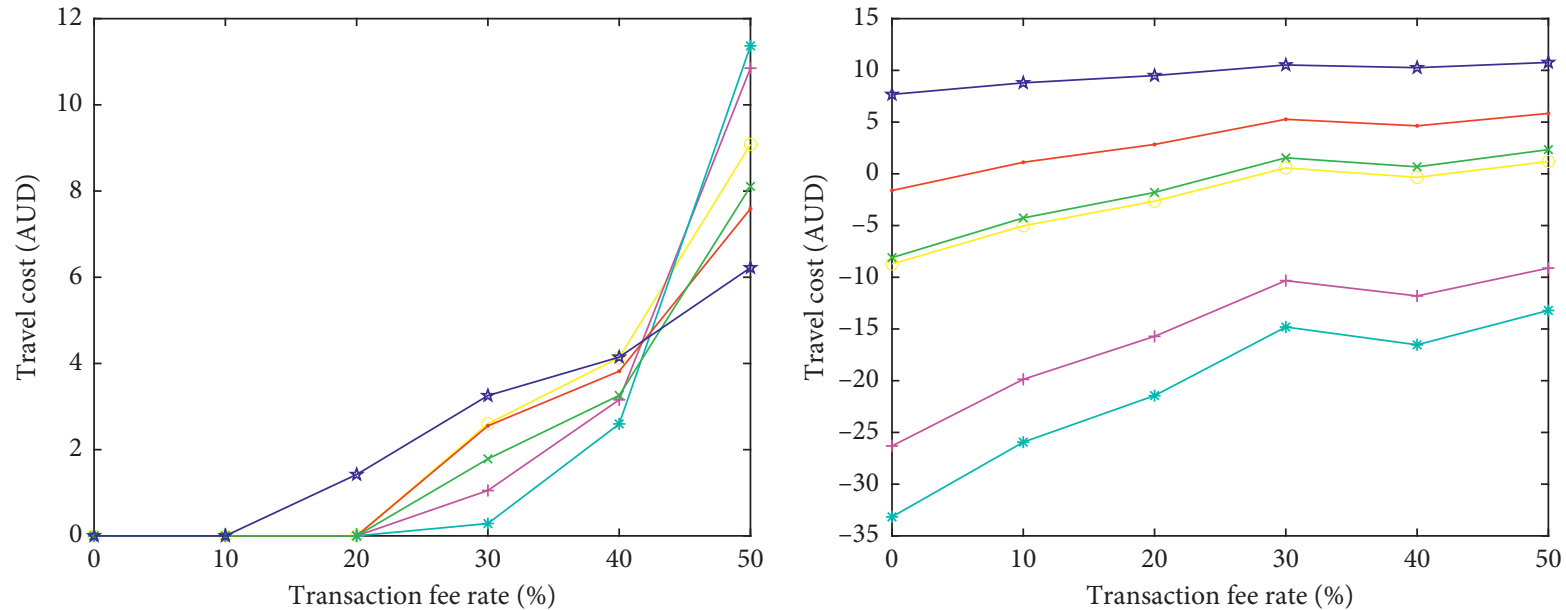

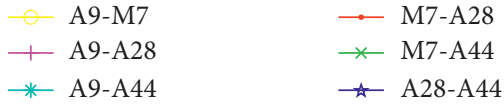

(c)

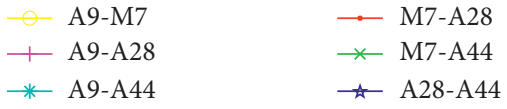

(d)

FIgURE 7: Detailed analysis about transaction fee rate for the 6 main ODs in the network. (a) Vehicle occupancy as a function of transaction fee rate. (b) Speed as a function of transaction fee rate. (c) Average RSP travel cost as a function of transaction fee rate. (d) Average RSD travel cost as a function of transaction fee rate.

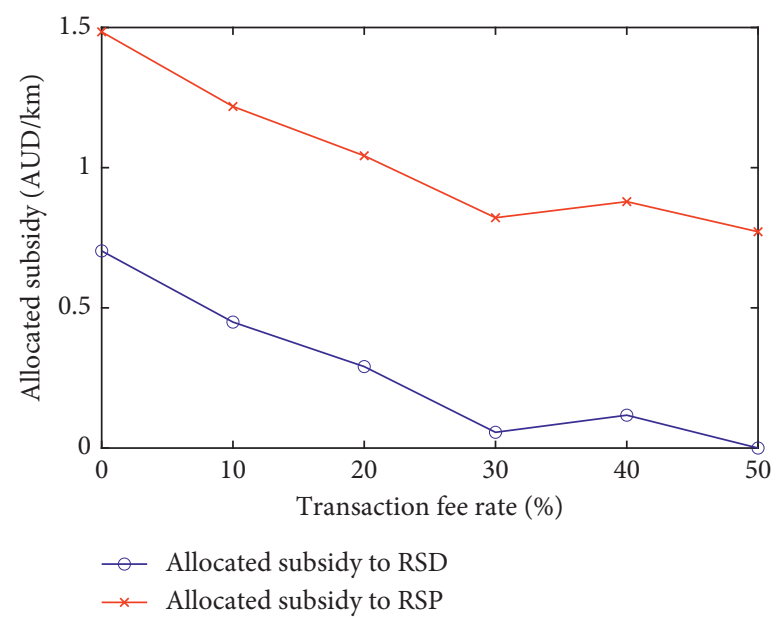

FIGURE 8: Subsidy allocated as a function of transaction fee rate. 


\section{Conclusion}

In this study, we have explored the potential of providing monetary incentives for ridesharing users, i.e., subsidies, to increase social welfare and reduce traffic congestion by increasing the vehicle occupancy rate. We focus on a freeway network with multiple origin-destination pairs and consider four travel modes including solo drivers, ridesharing drivers, ridesharing passengers, and a reserve option that does not contribute to congestion on the network. We proposed a simulation-based optimization framework that combines iterates between a global optimization heuristic that selects subsidies and a simulation procedure that aims to determine equilibrium mode choice proportions in the long run.

The proposed simulation procedure iterates between a link transmission model (LTM) to determine traffic congestion and calculating logit-compatible mode choice proportions. Upon convergence, the proposed simulation-based optimization approach finds equilibrated travel demand proportions across travel modes. We considered a realistic case study representative of the morning commute on Sydney's M4 Motorway in Australia, synthesized from open data sources.

Our numerical experiments reveal that the vehicle occupancy rate can be increased up to 2.4 on some OD of the freeway and that congestion levels can be substantially reduced by adequately setting the maximum subsidy available and the transaction fee rate between ridesharing passengers and drivers. For this, excessively increasing the maximum available subsidy to ridesharing users may be counterproductive from a congestion mitigation standpoint, thus providing quantifiable insight into the impacts of ridesharing incentives on social welfare and system performance. In addition, the subsidies to ridesharing drivers (RSD) and ridesharing passengers (RSP) could be implemented under different policies since the optimal subsidy to RSD to maximize welfare may not always be the maximum subsidy available. Overall, we find that subsidy-based ridesharing incentives may help in reducing the number of vehicles on the road, which could also lead to reduction in terms of environmental cost [64]. Furthermore, the approach proposed in this study may help informing on investment strategies across public transit (represented by the reserve option) and road infrastructure may benefit from the reduction of on-road vehicles. The transport facility is assumed invariable in the experiment. Nevertheless, since the findings of this study are obtained based on a case study of Sydney's M4 Motorway, knowledge transfer to other networks and regions should be done with care.

This study may be extended in several directions. First, many ridesharing platforms use dynamic transaction fee rates which influences users' preferences in the long run [65]. Hence, modeling profit-maximizing mobility service providers and the impact of competition on travel demand across modes is a promising avenue of research. Second, the proposed model can be enriched by including additional features such as tolled roads, dynamic parking costs, and HOV lanes. Last, the present study only focused on a freeway network. Exploring the behavior of ridesharing incentives in a general dynamic network and accounting for users' route choice is a challenging and promising extension of this work.

\section{Data Availability}

The data used in our paper can be obtained from open data sources referenced in the paper.

\section{Conflicts of Interest}

The authors declare that they have no conflicts of interest.

\section{References}

[1] M. Terrill and G. Institute, Stuck in traffic?: Road Congestion in Sydney and Melbourne, Grattan Institute, 2017, https://books. google.com.au/books?id=nn7FtAEACAAJ.

[2] Chester D. (2017), 'Victorian transport association state conference'. URL: http://minister.infrastructure.gov.au/ chester/speeches/2017/dcs007_2017.aspx.

[3] Bureau of Transport Statistics (2016), 'Nsw and Sydney Transport Facts 2016'..

[4] G. Corpuz, "Analysis of peak hour travel using the sydney household travel survey data," in Proceedings from the 29th Australasian Transport Research Forum, Gold Coast, Australia, September 2006.

[5] E. E. Agency, Are We Moving in the Right Direction?: Indicators on Transport and Environment Integration in the EU, European Communities, Brussels, Belgium, 2000.

[6] X. Wang, M. Dessouky, and F. Ordonez, "A pickup and delivery problem for ridesharing considering congestion," Transportation Letters, vol. 8, no. 5, pp. 259-269, 2016.

[7] D. Merugu, B. S. Prabhakar, and N. Rama, "An incentive mechanism for decongesting the roads: a pilot program in Bangalore," in Proceedings of ACM NetEcon Workshop, Princeton, NJ, USA, December 2009.

[8] D. Rey, V. V. Dixit, J.-L. Ygnace, and S. T. Waller, "An endogenous lottery-based incentive mechanism to promote offpeak usage in congested transit systems," Transport Policy, vol. 46, pp. 46-55, 2016.

[9] N. Agatz, A. Erera, M. Savelsbergh, and X. Wang, "Optimization for dynamic ride-sharing: a review," European Journal of Operational Research, vol. 223, no. 2, pp. 295-303, 2012.

[10] F. Ciari and K. W. Axhausen, "Choosing carpooling or car sharing as a mode: Swiss stated choice experiments," in Proceedings of TRB 91st Annual Meeting Compendium of Papers, pp. 12-4205, Transportation Research Board, Washington, DC, USA, January 2012.

[11] X. Liu, X. Yan, F. Liu, R. Wang, and Y. Leng, "A trip-specific model for fuel saving estimation and subsidy policy making of carpooling based on empirical data," Applied Energy, vol. 240, pp. 295-311, 2019.

[12] B. Caulfield, "Estimating the environmental benefits of ridesharing: a case study of Dublin," Transportation Research Part D: Transport and Environment, vol. 14, no. 7, pp. 527-531, 2009.

[13] A. Levofsky and A. Greenberg, "Organized dynamic ride sharing: the potential environmental benefits and the opportunity for advancing the concept," in Proceedings of Transportation Research Board 2001 Annual Meeting, pp. 711, Washington, DC, USA, January 2001.

[14] B. Yu, Y. Ma, M. Xue et al., "Environmental benefits from ridesharing: a case of beijing," Applied Energy, vol. 191, pp. 141-152, 2017. 
[15] D. Brownstone and T. F. Golob, "The effectiveness of ridesharing incentives," Regional Science and Urban Economics, vol. 22, no. 1, pp. 5-24, 1992.

[16] S. Shaheen, A. Cohen, M. Randolph, E. Farrar, R. Davis, and A. Nichols, Ridesharing (Carpooling and Vanpooling), Technical Report, Institute of Transportation Studies, Berkeley, CF, USA, 2019.

[17] R. Ma and H. M. Zhang, "The morning commute problem with ridesharing and dynamic parking charges," Transportation Research Part B: Methodological, vol. 106, pp. 345374, 2017.

[18] R. W. Willson and D. C. Shoup, "Parking subsidies and travel choices: assessing the evidence," Transportation, vol. 17, no. 2, pp. 141-157, 1990.

[19] Y. Liu and Y. Li, "Pricing scheme design of ridesharing program in morning commute problem," Transportation Research Part C: Emerging Technologies, vol. 79, pp. 156-177, 2017.

[20] J. Kwon and P. Varaiya, "Effectiveness of California's high occupancy vehicle (HOV) system," Transportation Research Part C: Emerging Technologies, vol. 16, no. 1, pp. 98-115, 2008.

[21] R. Wang, "Shaping carpool policies under rapid motorization: the case of Chinese cities," Transport Policy, vol. 18, no. 4, pp. 631-635, 2011.

[22] POOLE R.. (2020), 'The impact of hov and hot lanes on congestion in the United States'. URL: https://www.itf-oecd. org/impact-hov-and-hot-lanes-congestion-united-states.

[23] A. Simonetto, J. Monteil, and C. Gambella, "Real-time cityscale ridesharing via linear assignment problems," Transportation Research Part C: Emerging Technologies, vol. 101, pp. 208-232, 2019.

[24] C. Gambella, J. Monteil, A. Dekusar, S. Cabrero Barros, A. Simonetto, and Y. Lassoued, "A city-scale IoT-enabled ridesharing platform," Transportation Letters, vol. 12, no. 10, pp. 706-712, 2020.

[25] V. Pandey, J. Monteil, C. Gambella, and A. Simonetto, "On the needs for maas platforms to handle competition in ridesharing mobility," Transportation Research Part C: Emerging Technologies, vol. 108, pp. 269-288, 2019.

[26] N. Agatz, A. L. Erera, M. W. P. Savelsbergh, and X. Wang, "Dynamic ride-sharing: a simulation study in metro atlanta," Procedia-Social and Behavioral Sciences, vol. 17, pp. 532-550, 2011.

[27] K. Ghoseiri, Dynamic Rideshare Optimized Matching Problem, PhD Thesis, University of Maryland, College Park, ML, USA, 2012.

[28] Y. Wang, S. Winter, and M. Tomko, "Collaborative activitybased ridesharing," Journal of Transport Geography, vol. 72, pp. 131-138, 2018.

[29] X. Wang, N. Agatz, and A. Erera, "Stable matching for dynamic ride-sharing systems," Transportation Science, vol. 52, no. 4, pp. 850-867, 2018.

[30] A. Najmi, D. Rey, and T. H. Rashidi, "Novel dynamic formulations for real-time ride-sharing systems," Transportation Research Part E: Logistics and Transportation Review, vol. 108, pp. 122-140, 2017.

[31] A. Di Febbraro, E. Gattorna, and N. Sacco, "Optimization of dynamic ridesharing systems," Transportation Research Record: Journal of the Transportation Research Board, vol. 2359, no. 1, pp. 44-50, 2013.

[32] W. He, D. Li, T. Zhang, L. An, M. Guo, and G. Chen, "Mining regular routes from gps data for ridesharing recommendations," in Proceedings of the ACM SIGKDD International
Workshop on Urban Computing, pp. 79-86, Beijing, China, August 2012.

[33] O. F. Aydin, I. Gokasar, and O. Kalan, "Matching algorithm for improving ride-sharing by incorporating route splits and social factors," PLoS One, vol. 15, no. 3, p. e0229674, 2020.

[34] A. Braverman, J. G. Dai, X. Liu, and L. Ying, "Empty-car routing in ridesharing systems," Operations Research, vol. 67, no. 5, pp. 1437-1452, 2019.

[35] A. Braverman, J. G. Dai, X. Liu, and L. Ying, "Fluid-modelbased car routing for modern ridesharing systems," $A C M$ SIGMETRICS Performance Evaluation Review, vol. 45, no. 1, pp. 11-12, 2017.

[36] W. Herbawi and M. Weber, "Evolutionary multiobjective route planning in dynamic multi-hop ridesharing," in Evolutionary Computation in Combinatorial Optimization, P. Merz and J.-K. Hao, Eds., Springer, Berlin, Heidelberg, pp. 84-95, 2011.

[37] K. K. Dewan and I. Ahmad, "Carpooling: a step to reduce congestion," Engineering Letters, vol. 14, no. 1, pp. 61-66, 2007.

[38] H. Xu, F. Ordóñez, and M. Dessouky, "A traffic assignment model for a ridesharing transportation market," Journal of Advanced Transportation, vol. 49, no. 7, pp. 793-816, 2015.

[39] H. Xu, J.-S. Pang, F. Ordóñez, and M. Dessouky, "Complementarity models for traffic equilibrium with ridesharing," Transportation Research Part B: Methodological, vol. 81, pp. 161-182, 2015.

[40] X. Chen, C. Xiong, X. He, Z. Zhu, and L. Zhang, “Time-of-day vehicle mileage fees for congestion mitigation and revenue generation: a simulation-based optimization method and its real-world application," Transportation Research Part C: Emerging Technologies, vol. 63, pp. 71-95, 2016.

[41] X. Chen, L. Zhang, X. He, C. Xiong, and Z. Zhu, "Simulationbased pricing optimization for improving network-wide travel time reliability," Transportmetrica A: Transport Science, vol. 14, no. 1-2, pp. 155-176, 2018.

[42] J. Ekström, I. Kristoffersson, and N.-H. Quttineh, "Surrogatebased optimization of cordon toll levels in congested traffic networks," Journal of Advanced Transportation, vol. 50, no. 6, pp. 1008-1033, 2016.

[43] Z. Gu, S. T. Waller, and M. Saberi, "Surrogate-based toll optimization in a large-scale heterogeneously congested network," Computer-Aided Civil and Infrastructure Engineering, vol. 34, no. 8, pp. 638-653, 2019.

[44] X. He, X. Chen, C. Xiong, Z. Zhu, and L. Zhang, "Optimal time-varying pricing for toll roads under multiple objectives: a simulation-based optimization approach," Transportation Science, vol. 51, no. 2, pp. 412-426, 2017.

[45] T. Litman, Understanding Transport Demands and Elasticities: How Prices and Other Factors Affect Travel Behavior, The National Academies of Sciences, Engineering, and Medicine, Washington, DC, USA, 2012.

[46] D. Gujarati and D. Porter, Basic Econometrics, McGraw-Hill/ Irwin, New York, NY, USA, 2008.

[47] T. D. Schuster, J. Byrne, J. Corbett, and Y. Schreuder, "Assessing the potential extent of carsharing," Transportation Research Record: Journal of the Transportation Research Board, vol. 1927, no. 1, pp. 174-181, 2005.

[48] I. Yperman, S. Logghe, and B. Immers, "The link transmission model: an efficient implementation of the kinematic wave theory in traffic networks," in Proceedings of the 10th EWGT Meeting, pp. 122-127, Poznan Poland, September 2005. 
[49] Australian Bureau of Statistics (2016), 'Journey to work'. URL: https://auth.censusdata.abs.gov.au/webapi/jsf/ dataCatalogueExplorer.xhtml.

[50] Roads and Maritime Services (2018), 'Traffic Volume Viewer'. URL: $\quad$ https://www.rms.nsw.gov.au/about/corporatepublications/statistics/traffic-volumes/aadt-map/index. $\mathrm{html} \# / ? \mathrm{z}=6$.

[51] Roads and Maritime Services (2015), 'M4 Smart Motorway'..

[52] N. Holyoak and Y. M. Chang, "Peak spreading behaviour and model development," in DOI: 10.2495/UT060211 ConferenceURBAN TRANSPORT', 2006.

[53] M. Zeibots, "Before and after sydney's m4 motorway: did it make the city more sustainable," in Proceedings of National Conference on the State of Australian Cities, pp. 1-3, Parramatta, Australia, December 2003.

[54] Transport for N. S. W. (2020), 'Sydney motorway toll charges'. URL: https://www.rms.nsw.gov.au/sydney-motorways/tollcharges/.

[55] OpenStreetMap contributors (2017), 'Planet dump retrieved from https://planet.osm.org', https://www. openstreetmap.org.

[56] Roads and Maritime Services (2017), 'Motorway Design Guide: Capacity and Flow Analysis'. URL: https://www.rms.nsw.gov. $\mathrm{au} /$ business-industry/partners-suppliers/documents/motorwaydesign/motorway-design- guide-capacity-flow-analysis.pdf.

[57] Z. Xiong, D. Rey, T. Mao, H. Liu, V. V. Dixit, and S. T. Waller, "A three-stage framework for motorway travel time prediction," in Proceedings of 17th International IEEE Conference on Intelligent Transportation Systems (ITSC), pp. 816-821, IEEE, Qingdoa, China, October 2014.

[58] Australian Bureau of Statistics (2016), 'Census'. URL: https:// www.abs.gov.au/census.

[59] Bureau of Transport Statistics (2020), 'Average weekly earnings, Australia'. URL: https://www.abs.gov.au/statistics/ labour/earnings-and-work-hours/average-weekly-earningsaustralia/latest-release.

[60] The Department of Infrastructure, Transport, Regional Development and Communications (2020), 'Australian transport assessment and planning'. URL: https://www.atap.gov. $\mathrm{au} /$ parameter-values/road-transport/3-travel-time.

[61] D. A. HensherB. Wang et al., "Productivity foregone and leisure time corrections of the value of business travel time savings for land passenger transport in Australia," Road \& Transport Research: A Journal of Australian and New Zealand Research and Practice, vol. 25, no. 2, p. 15, 2016.

[62] W. Himpe, R. Corthout, and M. J. C. Tampère, "An efficient iterative link transmission model," Transportation Research Part B: Methodological, vol. 92, pp. 170-190, 2016.

[63] C. M. J. Tampère, R. Corthout, D. Cattrysse, and L. H. Immers, "A generic class of first order node models for dynamic macroscopic simulation of traffic flows," Transportation Research Part B: Methodological, vol. 45, no. 1, pp. 289-309, 2011.

[64] Centre for International Economics (2005), 'Sydney's Transport Infrastructure: The Real Economics'..

[65] S. Banerjee, R. Johari, and C. Riquelme, "Dynamic pricing in ridesharing platforms," ACM SIGecom Exchanges, vol. 15, no. 1, pp. 65-70, 2016. 